\title{
ERRATUM
}

\section{Sequential combination of troposphere time series}

\section{Keshin}

Published online: 29 September 2006

(C) Springer-Verlag 2006

\section{Erratum to: GPS Solut \\ DOI 10.1007/s10291-006-0028-6}

Unfortunately errors occurred in Eqs. 9 and 23. The correct equations are given below:

$$
\begin{aligned}
& E\left\{\left(\begin{array}{c}
\mathbf{y}_{i} \\
\mathbf{b}_{i, i-1}
\end{array}\right)\right\}=\left(\begin{array}{c}
A_{\mathbf{Y}} \\
0
\end{array}\right) \cdot \mathbf{Y}_{i}+\left(\begin{array}{c}
A_{\mathbf{b}} \\
I_{K K}
\end{array}\right) \cdot \mathbf{b}_{i} ; \\
& D\left\{\left(\begin{array}{c}
\mathbf{y}_{i} \\
\mathbf{b}_{i, i-1}
\end{array}\right)\right\}=\left(\begin{array}{cc}
Q_{\mathbf{y}_{i}} & 0 \\
0 & Q_{\mathbf{b}_{i, i-1}}
\end{array}\right) \\
& Q_{\mathbf{w}_{i, i-1}}=Q_{\mathbf{w}_{i-1}}+Q=Q_{\mathbf{w}_{i-1}}+\sigma_{\mathbf{w}}^{2} \Delta t \cdot I_{K K}
\end{aligned}
$$

The online version of the original article can be found at http://dx.doi.org/10.1007/s10291-006-0028-6.

M. Keshin ( $\square)$

Department of Earth Observation and Space Systems (DEOS), Delft University of Technology (TU Delft), Kluyverweg, 1, P.O. Box 5058, 2600 GB Delft,

The Netherlands

e-mail: M.O.Kechine@tudelft.nl 